\title{
Economic evidence for the prevention and treatment of atopic eczema: a protocol for a systematic review
}

Tracey Helen Sach ${ }^{*}$, Emma McManus $^{1}$, Christopher Mcmonagle $^{1}$ and Nick Levell ${ }^{2}$

\begin{abstract}
Background: Eczema, synonymous with atopic eczema or atopic dermatitis, is a chronic skin disease that has a similar impact on health-related quality of life as other chronic diseases. The proposed research aims to provide a comprehensive systematic assessment of the economic evidence base available to inform economic modelling and decision making on interventions to prevent and treat eczema at any stage of the life course. Whilst the Global Resource of Eczema Trials (GREAT) database collects together the effectiveness evidence for eczema, there is currently no such systematic resource on the economics of eczema. It is important to gain an overview of the current state of the art of economic methods in the field of eczema in order to strengthen the economic evidence base further.

Methods/design: The proposed study is a systematic review of the economic evidence surrounding interventions for the prevention and treatment of eczema. Relevant search terms will be used to search MEDLINE, EMBASE, Database of Abstracts of Reviews of Effects, Cochrane Database of Systematic Reviews, Cochrane Central Register of Controlled Trials, National Health Service (NHS) Economic Evaluation Database, Health Technology Assessment, Cumulative Index to Nursing and Allied Health Literature, EconLit, Scopus, Cost-Effectiveness Analysis Registry and Web of Science in order to identify relevant evidence. To be eligible for inclusion studies will be primary empirical studies evaluating the cost, utility or full economic evaluation of interventions for preventing or treating eczema. Two reviewers will independently assess studies for eligibility and perform data abstraction. Evidence tables will be produced presenting details of study characteristics, costing methods, outcome methods and quality assessment. The methodological quality of studies will be assessed using accepted checklists.

Discussion: The systematic review is being undertaken to identify the type of economic evidence available, summarise the results of the available economic evidence and critically appraise the quality of economic evidence currently available to inform future economic modelling and resource allocation decisions about interventions to prevent or treat eczema. We aim to use the review to offer guidance about how to gather economic evidence in studies of eczema and/or what further research is necessary in order to inform this.
\end{abstract}

Systematic review registration: PROSPERO CRD42015024633

Keywords: Eczema, Economics, Costs, Health-related quality of life, Cost effectiveness

\footnotetext{
*Correspondence: T.Sach@uea.ac.uk

'Health Economics Group, Norwich Medical School, University of East Anglia,

Norwich NR4 7TJ, UK

Full list of author information is available at the end of the article
} 


\section{Background}

In the UK, the lifetime prevalence of eczema is estimated to be between 16 and $20 \%$ making it the commonest inflammatory skin condition in children, and it has been increasing in "western style" environments [1-3]. In the UK, the age-sex standardised incidence and lifetime prevalence of eczema has increased between 2001 and 2005 from 9.58 per 1000 to 13.58 per 1000 patients and 77.78 per 1000 to 115.26 per 1000 , respectively [4]. Up to $50 \%$ of childhood cases will experience recurrence in adulthood [2]. Eczema is largely managed in primary care. Skin conditions are the commonest new reason patients consult their GP [5]. Eczema has been found to have a similar impact on health-related quality of life as other common childhood conditions such as asthma and diabetes [6]. Eczema impacts quality of life by causing itching, sleep loss and social stigma for the child. Families may also suffer from sleep loss and time taken off work to accompany children to health appointments [7]. The condition is associated with atopy so children with the condition are more likely to develop asthma and allergic rhinitis [8]. It is also believed that eczema has large cost implications. For instance, in 1995-1996, the total annual UK cost of eczema in children aged 5 years or younger was estimated as $£ 47$ million ( $£ 80$ per child) [9]. Looking at a broader age range, the UK total annual cost was estimated to be around $£ 465$ million, of which $£ 125$ million were National Health Service (NHS) costs, £297 million costs incurred by patients and $£ 42$ million by society in terms of lost productivity (price year not reported) [10]. These UK-based estimates of the total annual UK costs of eczema are now dated, the estimates were based on small samples [146 in [10] and 1523 in [9]], and the range of treatments available has increased and is likely to increase in the future with the addition of new biologics [11].

Despite eczema being common, there remain many unknowns about how to prevent and treat the condition. The James Lind Alliance (JLA) [12] Priority Setting Partnership (PSP) on eczema illustrates this (http://www.jla. nihr.ac.uk/priority-setting-partnerships/eczema and [13]). The JLA facilitates disease specific PSPs that bring together patients, carers and health professionals to identify and prioritise research for the treatment uncertainties of the disease of interest (http://www.jla.nihr.ac.uk/). The eczema PSP was established in 2010 with partners drawn from academic, NHS and charitable sectors and resulted in 14 treatment uncertainties being prioritised [13].

In order to draw together the effectiveness evidence of interventions for eczema, the Global Resource of Eczema Trials (GREAT) [14] database was established [15] and includes details of over 600 systematic reviews and randomised controlled trials. It does not, however, identify or bring together the economic literature on eczema, and thus, this review attempts to do this. There is likely to be less economic evidence, compared to effectiveness data, for eczema. Indeed, the English National Institute of Health and Care Excellence (NICE) has only considered economic models for two areas of eczema care: an educational intervention for those with eczema aged under 12 [16] and tacrolimus and pimecrolimus for atopic eczema [17]. It is, therefore, important to identify the current state of economic evidence addressing eczema in order to inform the design of future economic research in the area.

The proposed systematic review will address the following four research questions:

1. What type of health economic evidence has been used in the evaluation of the prevention and treatment of atopic eczema?

2. Are interventions to prevent and treat atopic eczema cost effective?

3. What is the quality of the health economic evidence for the prevention and treatment of atopic eczema?

4. What are the current gaps in the existing research?

\section{Methods/design \\ Protocol and registration}

The Preferred Reporting Items for Systematic Reviews and Meta-Analyses Protocols (PRISMA-P) statement recommendations were used to develop the methods for this systematic review (see Additional file 1) and will be used in reporting the results from the study [18]. This protocol has been registered in the International Prospective Register of Systematic reviews (PROSPERO) CRD42015024633. Should protocol amendments be necessary, these will be documented including details of the date, changes made and the rationale for changes.

\section{Literature search}

The following electronic databases will be searched: MEDLINE, EMBASE, Cumulative Index to Nursing and Allied Health Literature, Cochrane Central Register of Controlled Trials, Database of Abstracts of Reviews of Effects, Cochrane Database of Systematic Reviews, NHS Economic Evaluation Database, EconLit, Scopus, Health Technology Assessment, Cost-Effectiveness Analysis Registry and Web of Science. There will be no restriction imposed on the search from date; therefore, the literature search will go from the earliest records within each database, to the single day that the search was conducted on. Search results will be downloaded to Endnote version X7 where duplicates will be identified and removed.

Reference lists of potential eligible studies, reviews, guidelines or other sources will be screened for additional literature. Authors of published abstracts and 
conference proceedings will be contacted by email to establish if a full paper has since been published in the grey literature.

The search strategy was guided by The Cochrane Collaboration Handbook [19] and the Centre for Reviews and Dissemination guidelines for systematic reviews [20]. Specifically, search terms used in other atopic eczema systematic reviews were used to inform the clinical search terms [21]) and were also informed by the clinical author on the paper: NL. The economic terms were similarly devised, by consulting relevant guidelines $[22,23]$. The complete search strategy (with interface and coverage dates) is available in the Appendix to this protocol.

\section{Eligible studies}

A study will be included if it reports primary data on cost and/or outcome (utility or willingness to pay) data on atopic eczema. The primary interest is in full economic evaluations (cost effectiveness, cost utility, cost benefit and cost minimisation) although other partial economic evidence will also be included where the study has an explicit economic objective, this is likely to include cost-consequence analyses, cost analyses, utility assessment or willingness to pay/accept studies. There will be no restriction on the study designs used in the economic studies so, for example, economic studies conducted alongside randomised controlled trials, as part of observational studies, or as decision model-based analyses will be included. Nor will there be any restrictions on type of setting. The search was undertaken on the 9th October 2015, so only studies published before this date are included. Only full text articles published in the English language will be included, abstracts and letters will be excluded. Where two or more studies appear to be reporting on the same dataset or using the same model, the most comprehensive paper will be included unless each paper reports on a different aspect or in relation to a different jurisdiction/population (in the case of modelling studies).

\section{Data collection}

\section{Study selection}

Two independent reviewers will assess the titles and abstracts retrieved in the literature search against our inclusion criteria. In a second stage, full-text articles for those seeming to fit the criteria or where there is uncertainty about relevance will be retrieved and their eligibility assessed according to criteria set out in Table 1. Where disagreements occur a third reviewer will be involved.

\section{Data abstraction and management}

Data will be extracted independently by two reviewers and entered into an electronic data extraction form developed in Microsoft Excel, with the third reviewer consulted in case of disagreements that cannot be resolved between the two reviewers. A full list of the extraction fields can be found in Table 2. The data extraction form was piloted, modified (where necessary) and reviewers' responses calibrated on the basis of two pre-identified studies (one modelling study and one non-model-based paper). The data extracted will be analysed in a narrative/descriptive manner focusing on the methods, results and quality of studies included with the aim of identifying gaps in the evidence, areas of strength and areas in need of methodological improvement.

If the necessary data are available, the results will be discussed as subsets for different age groups (e.g. child/ adult/elderly) and/or different skin disease severities and/or world regions and/or health-care settings. Furthermore, as it is expected that included studies will report results in a range of currencies, where possible, results will be reported in the original currency and price year, as well as being converted to UK pounds using the purchasing power parities provided by the Organisation for Economic Co-operation and Development (OECD), inflating to a common price year using the consumer price index, to facilitate the comparison of results across studies.

Table 1 Inclusion and exclusion criteria

\begin{tabular}{|c|c|c|}
\hline & Inclusion criteria & Exclusion criteria \\
\hline \multirow[t]{2}{*}{ Population } & \multirow{2}{*}{$\begin{array}{l}\text { Eczema (synonyms: atopic eczema, atopic dermatitis) ICD-10 } \\
\text { code: L20 }\end{array}$} & Populations with other skin diseases other than eczema \\
\hline & & $\begin{array}{l}\text { Dyshidrotic eczema, seborrheic eczema, chronic actinic } \\
\text { dermatitis, asteatotic eczema, allergic contact eczema, irritant } \\
\text { contact eczema, varicose eczema, stasis eczema ICD-10 codes: } \\
\text { L21 to L30 }\end{array}$ \\
\hline \multirow[t]{2}{*}{ Study designs } & \multirow{2}{*}{$\begin{array}{l}\text { Studies presenting primary data in the form of a Cost of illness, } \\
\text { cost effectiveness, cost utility, cost benefit, cost consequences, } \\
\text { cost analysis, utility analysis, contingent valuation }\end{array}$} & Studies without an explicit economic objective \\
\hline & & $\begin{array}{l}\text { Review articles, although the reference list for these will be } \\
\text { checked for primary studies that should be included }\end{array}$ \\
\hline Outcomes & Utility, QALYs, willingness to pay/accept & \\
\hline Publication type & Articles available in full text in English & Abstracts, letters, reviews \\
\hline
\end{tabular}


Table 2 Data abstraction fields

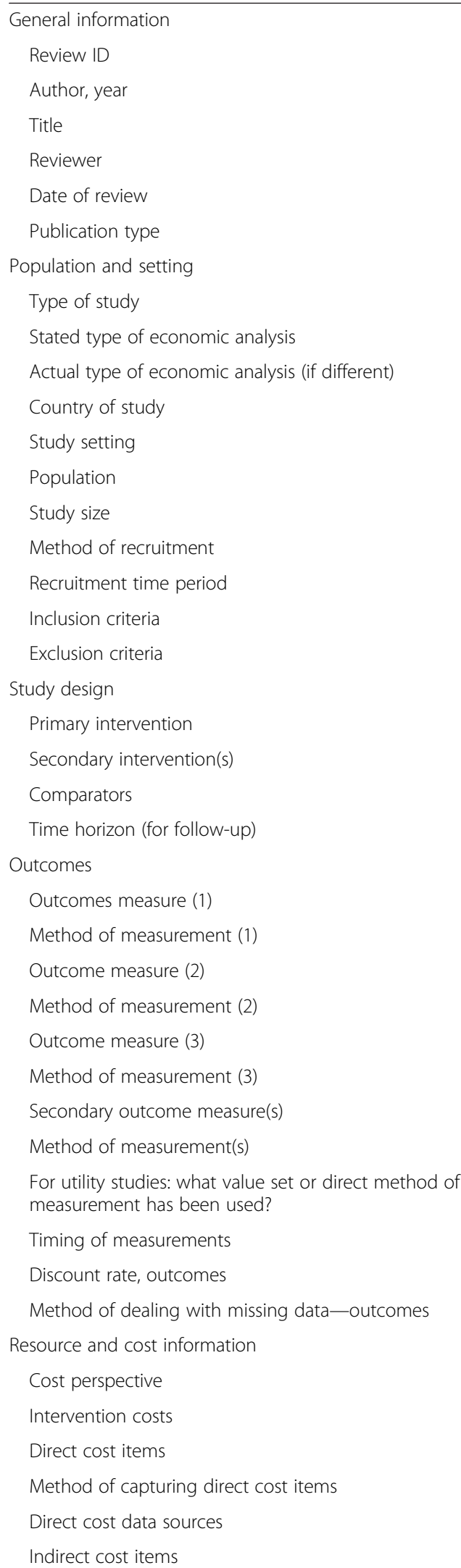

Table 2 Data abstraction fields (Continued)

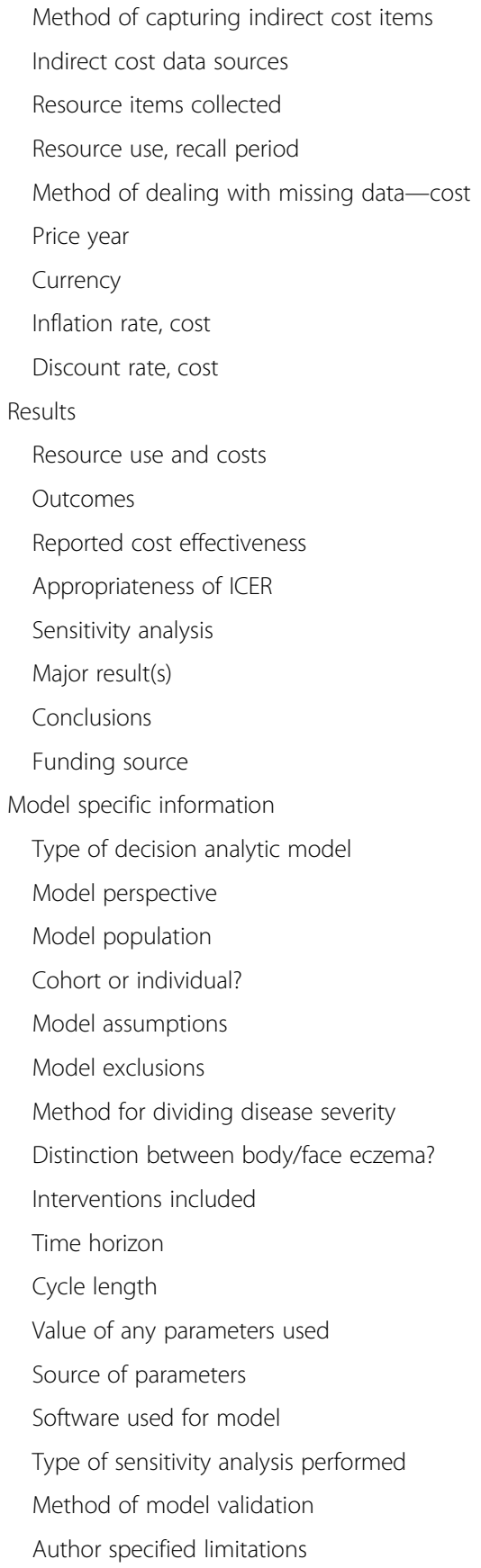

\section{Quality assessment and data presentation}

Two reviewers will independently evaluate the quality of included studies in order to assess the risk of bias. Studies will be assessed using a published checklist based on a modified version of the Consolidated Health Economic Evaluation Reporting Standards (CHEERS) framework [24] (see Table 3). In addition, model-based economic evaluations will also be assessed using the Phillips criterion $[25,26]$ (see Table 4 for extraction 
Table 3 CHEERS checklist [24]

Item no. Recommendation $\quad$ Reported on page/ Yes/no/partial/unclear/

Title and abstract

Title

Abstract

Introduction

Background and objectives

Methods

Target population and subgroups

Setting and location

Study perspective

Comparators

Time horizon

Discount rate

Choice of health outcomes

Measurement of effectiveness

Measurement and valuation of preference based outcomes

Estimating resources and costs
Identify the study as an economic evaluation or use more specific terms such as "cost effectiveness analysis", and describe the interventions compared.

2 Provide a structured summary of objectives, perspective, setting, methods (including study design and inputs), results (including base case and uncertainty analyses), and conclusions.

Provide an explicit statement of the broader context for the study. Present the study question and its relevance for health policy or practice decisions.

Describe characteristics of the base case population and subgroups analysed, including why they were chosen.

5 State relevant aspects of the system(s) in which the decision(s) need(s) to be made.

6 Describe the perspective of the study and relate this to the costs being evaluated.

Describe the interventions or strategies being compared and state why they were chosen State the time horizon(s) over which costs and consequences are being evaluated and say why appropriate.

Report the choice of discount rate(s) used for costs and outcomes and say why appropriate.

10 Describe what outcomes were used as the measure(s) of benefit in the evaluation and their relevance for the type of analysis performed.

11a Single study-based estimates: Describe fully the design features of the single effectiveness study and why the single study was a sufficient source of clinical effectiveness data.

$11 \mathrm{~b} \quad$ Synthesis-based estimates: Describe fully the methods used for identification of included studies and synthesis of clinical effectiveness data

12 If applicable, describe the population and methods used to elicit preferences for outcomes.

13a Single study-based economic evaluation: Describe approaches used to estimate resource use associated with the alternative interventions. Describe primary or secondary research methods for valuing each resource item in terms of its unit cost. Describe any adjustments made to approximate to opportunity costs.

13b Model-based economic evaluation: Describe approaches and data sources used to estimate resource use associated with model health states. Describe primary or secondary research methods for valuing each resource item in terms of its unit cost. Describe any adjustments made to approximate to opportunity costs. 
Table 3 CHEERS checklist [24] (Continued)

Currency, price date, and conversion

Choice of model

Assumptions

Analytical methods

Results

Study parameters

Incremental costs and outcomes

Characterising uncertainty

Characterising heterogeneity

Discussion

Study findings, limitations, generalisability, and current knowledge

Other

Source of funding

Conflicts of interest
14

Report the dates of the estimated resource quantities and unit costs. Describe methods for adjusting estimated unit costs to the year of reported costs if necessary. Describe methods for converting costs into a common currency base and the exchange rate.

15 Describe and give reasons for the specific type of decision analytical model used. Providing a figure to show model structure is strongly recommended.

16 Describe all structural or other assumptions underpinning the decision-analytical model.

17 Describe all analytical methods supporting the evaluation. This could include methods for dealing with skewed, missing, or censored data; extrapolation methods; methods for pooling data; approaches to validate or make adjustments (such as half cycle corrections) to a model; and methods for handling population heterogeneity and uncertainty.

8 Report the values, ranges, references, and, if used probability distributions for all parameters. Report reasons or sources for distributions used to represent uncertainty where appropriate. Providing a table to show the input values is strongly recommended.

For each intervention, report mean values for the main categories of estimated costs and outcomes of interest as well as mean differences between the comparator groups. If applicable, report incremental cost effectiveness ratios.

20a Single study-based economic evaluation: Describe the effects of sampling uncertainty for the estimated incremental cost and incremental effectiveness parameters, together with the impact of methodological assumptions (such as discount rate, study perspective).

20b Model-based economic evaluation: Describe the effects on the results of uncertainty for all input parameters, and uncertainty related to the structure of the model and assumptions.

21 If applicable, report differences in costs, outcomes, or cost effectiveness that can be explained by variations between subgroups of patients with different baseline characteristics or other observed variability in effects that are not reducible by more information.

22 Summarise key study findings and describe how they support the conclusions reached. Discuss limitations and the generalisability of the findings and how the findings fit with current knowledge.

23 Describe how the study was funded and the role of the funder in the identification, design, conduct, and reporting of the analysis. Describe other non-monetary sources of support.

24 Describe any potential for conflict of interest of study contributors in accordance with journal policy. In the absence of a journal policy, we recommend authors comply with International Committee of Medical Journal Editors recommendations 
Table 4 Philips criterion [25, 26]

\begin{tabular}{|c|c|c|c|}
\hline Dimensions of quality & Questions for critical appraisal & $\begin{array}{l}\text { Response (Yes/no/ } \\
\text { partial/unclear/NA) }\end{array}$ & Comments \\
\hline \multirow[t]{3}{*}{ S1: Statement of decision problem/objective } & Is there a clear statement of the decision problem? & & \\
\hline & $\begin{array}{l}\text { Is the objective of the evaluation and model specified and } \\
\text { consistent with the stated decision problem? }\end{array}$ & & \\
\hline & Is the primary decision maker specified? & & \\
\hline \multirow[t]{4}{*}{ S2: Statement of scope/perspective } & Is the perspective of the model stated clearly? & & \\
\hline & Are the model inputs consistent with the stated perspective? & & \\
\hline & Has the scope of the model been stated and justified? & & \\
\hline & $\begin{array}{l}\text { Are the outcomes of the model consistent with the perspective, } \\
\text { scope and overall objective of the model? }\end{array}$ & & \\
\hline \multirow[t]{5}{*}{ S3: Rationale for structure } & Has the evidence regarding the model structure been described? & & \\
\hline & $\begin{array}{l}\text { Is the structure of the model consistent with a coherent theory of } \\
\text { the health condition under evaluation? }\end{array}$ & & \\
\hline & $\begin{array}{l}\text { Have any competing theories regarding model structure been } \\
\text { considered? }\end{array}$ & & \\
\hline & $\begin{array}{l}\text { Are the sources of data used to develop the structure of the } \\
\text { model specified? }\end{array}$ & & \\
\hline & $\begin{array}{l}\text { Are the causal relationships described by the model structure } \\
\text { justified appropriately? }\end{array}$ & & \\
\hline \multirow[t]{2}{*}{ S4: Structural assumptions } & Are the structural assumptions transparent and justified? & & \\
\hline & $\begin{array}{l}\text { Are the structural assumptions reasonable given the overall } \\
\text { objective, perspective and scope of the model? }\end{array}$ & & \\
\hline \multirow[t]{3}{*}{ S5: Strategies/comparators } & Is there a clear definition of the options under evaluation? & & \\
\hline & Have all feasible and practical options been evaluated? & & \\
\hline & Is there justification for the exclusion of feasible options? & & \\
\hline S6: Model type & $\begin{array}{l}\text { Is the chosen model type appropriate given the decision problem } \\
\text { and specified causal relationships within the model? }\end{array}$ & & \\
\hline \multirow[t]{3}{*}{ S7: Time horizon } & $\begin{array}{l}\text { Is the time horizon of the model sufficient to reflect all important } \\
\text { differences between options? }\end{array}$ & & \\
\hline & $\begin{array}{l}\text { Is the time horizon of the model, and the duration of treatment } \\
\text { and treatment effect described and justified? }\end{array}$ & & \\
\hline & $\begin{array}{l}\text { Has a lifetime horizon been used? If not, has a shorter time } \\
\text { horizon been justified? }\end{array}$ & & \\
\hline S8: Disease states/pathways & $\begin{array}{l}\text { Do the disease states (state transition model) or the pathways } \\
\text { (decision tree model) reflect the underlying biological process of } \\
\text { the disease in question and the impact of interventions? }\end{array}$ & & \\
\hline S9: Cycle length & $\begin{array}{l}\text { Is the cycle length defined and justified in terms of the natural } \\
\text { history of disease? }\end{array}$ & & \\
\hline \multirow[t]{6}{*}{ D1: Data identification } & $\begin{array}{l}\text { Are the data identification methods transparent and appropriate } \\
\text { given the objectives of the model? }\end{array}$ & & \\
\hline & $\begin{array}{l}\text { Where choices have been made between data sources, are these } \\
\text { justified appropriately? }\end{array}$ & & \\
\hline & $\begin{array}{l}\text { Has particular attention been paid to identifying data for the } \\
\text { important parameters in the model? }\end{array}$ & & \\
\hline & $\begin{array}{l}\text { Has the process of selecting key parameters been justified and } \\
\text { systematic methods used to identify the most appropriate data? }\end{array}$ & & \\
\hline & Has the quality of the data been assessed appropriately? & & \\
\hline & $\begin{array}{l}\text { Where expert opinion has been used, are the methods described } \\
\text { and justified? }\end{array}$ & & \\
\hline
\end{tabular}


Table 4 Philips criterion $[25,26]$ (Continued)

D2: Pre-model data

D2a: Baseline data

D2b: Treatment effects

D2c: Quality-of-life weights (utilities)

D3: Data incorporation

D4: Assessment of uncertainty

D4a: Methodological

D4b: Structura

D4c: Heterogeneity

D4d: Parameter

C1: Internal consistency

C2: External consistency
Are the pre-model data analysis methodology based on justifiable statistical and epidemiological techniques?

Is the choice of baseline data described and justified?

Are transition probabilities calculated appropriately?

Has a half cycle correction been applied to both cost and outcome?

If relative treatment effects have been derived from trial data, have they been synthesised using appropriate techniques?

Have the methods and assumptions used to extrapolate shortterm results to final outcomes been documented and justified? Have alternative assumptions been explored through sensitivity analysis?

Have assumptions regarding the continuing effect of treatment once treatment is complete been documented and justified? Have alternative assumptions been explored through sensitivity analysis?

Are the utilities incorporated into the model appropriate?

Is the source for the utility weights referenced?

Are the methods of derivation for the utility weights justified?

Have all data incorporated into the model been described and referenced in sufficient detail?

Has the use of mutually inconsistent data been justified (i.e. are assumptions and choices appropriate)?

Is the process of data incorporation transparent?

If data have been incorporated as distributions, has the choice of distribution for each parameter been described and justified?

Have the four principal types of uncertainty been addressed?

If not, has the omission of particular forms of uncertainty been justified?

Have methodological uncertainties been addressed by running alternative versions of the model with different methodological assumptions?

Is there evidence that structural uncertainties have been addressed via sensitivity analysis?

Has heterogeneity been dealt with by running the model separately for different sub-groups?

Are the methods of assessment of parameter uncertainty appropriate?

Has probabilistic sensitivity analysis been done, if not has this been justified?

If data are incorporated as point estimates, are the ranges used for sensitivity analysis stated and justified?

Is there evidence that the mathematical logic of the model has been tested thoroughly before use?

Are the conclusions valid given the data presented?

Are any counterintuitive results from the model explained and justified?

If the model has been calibrated against independent data, have any differences been explained and justified?

Have the results of the model been compared with those of previous models and any differences in results explained? 
table). Any discrepancies will be discussed and resolved by a third reviewer.

To initially assess the quality of included studies, a score of 1 will be given to items within the CHEERS checklist that can be answered 'yes', 0.5 for items only partially addressed, 0 for 'no' and where an item is identified as not applicable, no score will be given. The score will then be totalled and calculated as a percentage of the total score that could be achieved for that study, thus taking into account elements of the checklist which were deemed not applicable. This is an approach that has previously been used [27], and although it is acknowledged that by assigning equal weighting to each criteria may not truly reflect the importance of each of the checklist items, it is thought that it will provide a broad overview of the quality of studies included, which will then pave way for more detailed analysis on individual checklist items. These evaluations will be included in any publication as supplementary material where feasible.

Methodological variation between studies is likely to prevent a pooling of economic data in the form of a meta-analysis, and therefore, results of the studies will be presented and discussed in a qualitative manner according to the study type.

\section{Discussion}

This systematic review will provide a comprehensive assessment of the type and quality of economic research used in the evaluation of interventions to prevent and treat eczema. The results of the review are likely to be written up in multiple publications, one focusing on an overview of the state of the art with additional papers focusing in more detail on particular methodologically aspects (for instance, the methods used in modelling studies). The review will report the range of costeffectiveness estimates found for interventions to prevent and treat atopic eczema, which may be useful in informing clinicians and decision makers about the relative value of different interventions for eczema and enable the value of eczema interventions to be compared with the cost effectiveness for other interventions in other disease areas. That is, it may help decision makers, on the basis of current information (if sufficient), to be able to answer questions about how to allocate limited resources between eczema and other disease areas and once allocated to eczema how to use those limited resources efficiently to maximise outcomes from eczema care. The review will also be of interest to methodologists interested in the range and quality of economic studies in this clinical field. Finally, this systematic review will help identify gaps in the current evidence base surrounding the economics of eczema to inform further research efforts in this area.

\section{Appendix}

Details of electronic bibliographic database search strings Medline (1946 to present) \& Embase (1974 to present)

(via Ovid, http://ovidsp.tx.ovid.com/)

1. (dermatitis or eczema).tw.

2. exp Eczema/

3. exp Dermatitis, Atopic/

4. exp Economics/

5. exp Quality-Adjusted Life Years/

6. exp Health Care Costs/

7. (econ* or cost* or price" or expenditure* or "pharmacoecon*" or budget* or "value of life" or qaly* or "quality adj* life year*" or utilit*" or pricing* or "net benefit" or "monetary benefit*" or "net health benefit"" or "willingness to pay" or "fee" or "charge"

8. 1 or 2 or 3

9. 4 or 5 or 6 or 7

10. 8 and 9

Web of Science (various indexes included earliest start date 1970 to present) (http://apps.webofknowledge.com)

1. $\mathrm{TS}=($ dermatitis or eczema)

2. TS=((econ* or cost* or price* or expenditure* or "pharmacoecon" ${ }^{*}$ or budget" or "value of life" or qaly" or "quality adj" life year*" or utilit" or pricing" or "net benefit" or "monetary benefit" or "net health benefit" or "willingness to pay" or "fee"*" or "charge*"))

3. 1 and 2

EconLit (1886 to present) and CINAHL (1937 to present) (via EBSCO, http://web.a.ebscohost.com)

1. MH "Dermatitis, Atopic" OR MH "Eczema"

2. AB Dermatitis OR Eczema

3. (MH "Economics+") OR (MH "Health Resource Allocation") OR (MH "Health Care Costs+") OR (MH "Health Care Delivery+") OR (MH "Quality Adjusted Life Years") OR (MH "Quality of Life") OR (MH "Economic Value of Life")

4. $\mathrm{AB}$ econ* or cost" or price" or expenditure* or "pharmacoecon" or budget" or "value of life" or qaly" or "quality adj" life year" or utilit" or pricing" or "net benefit" or "monetary benefit" or "net health benefit*" or "willingness to pay" or "fee"*" or "charge "*"

5. 1 or 2

6. 3 or 4

7. 5 and 6

Scopus (1960 to present) (http://www.scopus.com) 
1. (TITLEABSKEY (dermatitis OR eczema)) AND (TITLEABSKEY ((econ* OR cost* OR price* OR pricing* OR expenditure* OR "pharmacoecon*" OR budget" OR "value of life" OR qaly* OR "quality adj* life year*" OR utilit* OR "net benefit*" OR "monetary benefit*" OR "net health benefit*" OR "willingness to pay" OR "fee*" OR "charge*")))

Cochrane Database of Systematic Reviews/Database of Abstracts of Reviews of Effect/Cochrane Central Register of Controlled Trials/Health Technology Assessment Database/NHS Economic Evaluation Database (all years searched)

(via Wiley, http://onlinelibrary.wiley.com/cochranelibrary/ search/)

1. dermatitis or eczema.ti.ab

2. MeSH descriptor: [Dermatitis, Atopic] explode all trees OR MeSH descriptor: [Eczema] explode all trees

3. econ* or cost" or price* or expenditure* or "pharmacoecon*" or budget" or "value of life" or qaly" or "quality adj;" life year*" or utilit* or pricing* or "net benefit" or "monetary benefit" ${ }^{* *}$ or "net health benefit"" or "willingness to pay" or "fee" "charge" "ti.ab.

4. MeSH descriptor: [Economics] explode all trees OR MeSH descriptor: [Quality Adjusted Life Years] explode all trees OR MeSH descriptor: [Health Care Costs] explode all trees

5. 1 or 2

6. 3 or 4

7. 5 and 6

CEA Registry (1976 to present) (https://research. tufts-nemc.org/cear4/Home.aspx)

1. Eczema

2. Dermatitis

\section{Additional file}

Additional file 1: Preferred Reporting Items for Systematic review and Meta-Analysis Protocols (PRISMA-P) 2015 checklist: recommended items to address in a systematic review protocol. (DOCX 15kb)

\section{Abbreviations}

CHEERS: Consolidated Health Economic Evaluation Reporting Standards; GP: General Practitioner; GREAT: Global Resource for Eczema Trials; HQoL: Health-Related Quality of Life; ICER: Incremental Cost Effectiveness Ratio; JLA: James Lind Alliance; NA: Not Applicable; NHS: National Health Service; NICE: National Institute of Health and Care Excellence; PSP: Priority Setting Partnership; QALYs: Quality Adjusted Life Years; UK: United Kingdom.

\section{Acknowledgements}

This systematic review is independent research arising from Tracey Sach's Career Development Fellowship (CDF-2014-07-006) supported by the
National Institute for Health Research. The views expressed in this publication are those of the author(s) and not necessarily those of the NHS, the National Institute for Health Research or the Department of Health. The following individuals are acknowledged as providing advice as research mentors (Professor Kim Thomas, Dr Nicky Welton, Professor Andrew Briggs) and as part of the annual advisory panel (Dr Tom Kenny, Dr Nick Levell, Fiona McOwan, Jo Parris, Amanda Roberts, Professor Lee Shepstone, Professor Fujian Song and Dr Edward Wilson).

\section{Authors' contributions}

TS conceptualised the research plan for the proposed systematic review and wrote the manuscript. EM and CM contributed to the writing of the manuscript. NL provided expertise on eczema. All authors reviewed the manuscript for important intellectual content and approved the final manuscript. TS is the guarantor.

\section{Competing interests}

The authors declare that they have no competing interests.

\section{Author details}

${ }^{1}$ Health Economics Group, Norwich Medical School, University of East Anglia, Norwich NR4 7TJ, UK. ²Dermatology Department, Norfolk and Norwich University Hospitals NHS Foundation Trust, Colney Lane, Norwich NR4 7UY, UK.

Received: 12 January 2016 Accepted: 10 May 2016

Published online: 27 May 2016

\section{References}

1. Williams H, Stewart A, von Mutius E, Cookson W, Anderson HR. International study of asthma. Is eczema really on the increase worldwide? J Allergy Clin Immunol. 2008;121(4):947-54.

2. Williams HC. Clinical practice. Atopic dermatitis. N Engl J Med. 2005;352(22):2314-24.

3. Kay J, Gawkrodger DJ, Mortimer MJ, Jaron AG. The prevalence of childhood atopic eczema in a general population. J Am Acad Dermatol. 1994;30(1):35-9.

4. Simpson CR, Netwon J, Hippisley-Cox J, Sheikh A. Trends in the epidemiology and prescribing of medication for eczema in England. R Soc Med. 2009; doi:10.1258/jrsm.2009.080211.

5. Schofield JK, Fleming D, Grindlay D, Williams H. Skin conditions are the commonest new reason people present to general practitioners in England and Wales. Br J Dermatol. 2011;165(5):1044-50.

6. Lewis-jones S. Quality of life and childhood atopic dermatitis: the misery of living with childhood eczema. Int J Clin Pract. 2006;60(8):984-92.

7. Paller AS, Mcalister RO, Doyle JJ, Jackson A. Perceptions of physicians and pediatric patients about atopic dermatitis, its impact, and its treatment. Clin Paediatr. 2002;41(5):323-32.

8. Hon KLE, Wang SS, Leung TF. The atopic march: from skin to the airways. Iran J Allergy Asthma Immunol. 2012;11(1):73-7.

9. Emerson RM, Williams $\mathrm{HC}$, Allen BR. What is the cost of atopic dermatitis in preschool children? Br J Dermatol. 2001;144(3):514-22.

10. Herd RM, Tidman MJ, Prescott RJ, Hunter JAA. The cost of atopic eczema. Br J Dermatol. 1996;135(1):20-3.

11. Howell MD, Parker ML, Mustelin T, Ranade K. Past, present, and future for biologic intervention in atopic dermatitis. Allergy. 2015.

12. James Lind Alliance. Priority setting partnership on eczema. National Institute for Health Research. http://www.jla.nihr.ac.uk/priority-settingpartnerships/eczema Accessed 15 Oct 2015

13. Batchelor JM, Ridd MJ, Clarke T, Ahmed A, Cox M, Crowe S, et al. The eczema priority setting partnership: a collaboration between patients, carers, clinicians and researchers to identify and prioritize important research questions for the treatment of eczema. Br J Dermatol. 2013;168(3):577-82.

14. The Global Resource of Eczema Trials. Centre of evidence based dermatology. http://www.greatdatabase.org.uk. Accessed 05 Sep 2015.

15. Nankervis H, Maplethorpe A, Williams HC. Mapping randomized controlled trials of treatments for eczema-the GREAT database (The Global Resource of Eczema Trials: a collection of key data on randomized controlled trials of treatments for eczema from 2000 to 2010). BMC Dermatol. 2011;11(1):10.

16. NICE. Clinical Guideline 57: Atopic eczema in children-management of atopic eczema in children from birth up to the age of 12 years. 2007. 
Accessed online on 10th December 2015 at: http:/www.nice.org.uk/ guidance/cg57/evidence/full-guideline-196612237.

17. NICE. Tacrolimus and pimecrolimus for atopic eczema. NICE technology appraisal guidance [TA82]. 2004. Accessed online on 10th December 2015 at: http://www.nice.org.uk/guidance/TA82.

18. Shamseer L, Moher D, Clarke M, Ghersi D, Liberati A, Petticrew M, et al. Preferred reporting items for systematic review and meta-analysis protocols (PRISMA-P) 2015: elaboration and explanation. BMJ. 2015;349:g7647.

19. Higgins JPT, Green S (editors). Cochrane Handbook for Systematic Reviews of Interventions Version 5.1.0 [updated March 2011]. The Cochrane Collaboration, 2011. Available from http://handbook.cochrane.org

20. Centre for reviews and dissemination (CRD). Systematic reviews: CRD's guidance for undertaking reviews in health care. Centre for Reviews and Dissemination. 2009.

21. Futamura M, Thomas KS, Grindlay DJ, Doney EJ, Torley D, Williams HC Mapping systematic reviews on atopic eczema - an essential resource for dermatology professionals and researchers. PLoS ONE. 2013;8(3):58484.

22. Glanville J, Fleetwood K, Yellowlees A, Kaunelis D, Mensinkai S. Development and testing of search filters to identify economic evaluations in MEDLINE and EMBASE. Ottawa: Canadian Agency for Drugs and Technologies in Health; 2009.

23. McMaster University: Hedges. http://hiru.mcmaster.ca/hiru/HIRU_Hedges_ home.aspx (2016). Accessed 06 Apr 2016.

24. Husereau D, Drummond M, Petrou S, Carswell C, Moher D, Greenberg D, et al. Consolidated health economic evaluation reporting standards (CHEERS) statement. BMC Med. 2013;11(1):80

25. Philips Z, Ginnelly L, Sculpher M, Claxton K, Golder S, Riemsma R, et al. Review of guidelines for good practice in decision-analytic modelling in health technology assessment. Health Technol Assess. 2004;8:1-172.

26. Philips Z, Bojke L, Sculpher M, Claxton K, Golder S. Good practice guidelines for decision-analytic modelling in health technology assessment: a review and consolidation of quality assessment. PharmacoEconomics. 2006;24(4):355-71.

27. Buchanan J, Wordsworth $\mathrm{S}$. Welfarism versus extra-welfarism: can the choice of economic evaluation approach impact on the adoption decisions recommended by economic evaluation studies? PharmacoEconomics. 2015;33(6):571-9.

\section{Submit your next manuscript to BioMed Central and we will help you at every step:}

- We accept pre-submission inquiries

- Our selector tool helps you to find the most relevant journal

- We provide round the clock customer support

- Convenient online submission

- Thorough peer review

- Inclusion in PubMed and all major indexing services

- Maximum visibility for your research

Submit your manuscript at www.biomedcentral.com/submit

) Biomed Central 